\title{
Identificação de fatores de riscos junto a terapeutas ocupacionais no atendimento de portadores de disfunções físicas*
}

\section{Identification of factors of risks in occupational therapists in the attendance of carriers of physical dysfunctions}

\author{
Juliana Pires Cruz ${ }^{1}$, Nilson Rogério da Silva ${ }^{2}$
}

CRUZ, J. P.; SILVA, N. R. Identificação de fatores de riscos junto a terapeutas ocupacionais no atendimento de portadores de disfunções físicas. Rev. Ter. Ocup. Univ. São Paulo, v. 18, n. 1, p. 22-29, jan./abr., 2007.

\begin{abstract}
RESUMO: Dados do Ministério da Saúde (BRASIL, 2001) revelam índices crescentes de doenças ocupacionais em todos os segmentos profissionais. No setor da saúde, o profissional envolvido com o cuidado humano, também tem apresentado altos índices de doenças decorrentes do exercício profissional. Esta pesquisa buscou investigar as condições de trabalho de terapeutas ocupacionais que atuam junto a portadores de disfunções físicas visando identificar fatores de risco no trabalho e possíveis impactos na saúde dos mesmos, bem como propor medidas de prevenção e melhoria das situações de trabalho e consequentemente na assistência aos pacientes. A amostra foi composta por 10 terapeutas ocupacionais de Bauru e região. Os dados foram coletados a partir de um roteiro de entrevista estruturado e após a coleta transcritos integralmente, categorizados e organizados em figuras e tabelas. Foi possível concluir que as condições de trabalho oferecidas podem conduzir ao processo de adoecimento dos terapeutas ocupacionais. As atividades são realizadas predominantemente na postura em pé e sentada, com adoção de posturas inadequadas da coluna associado ao uso de força durante manuseio de pacientes; baixo índice de adesão às atividades de condicionamento físico, realizam pausas durante o trabalho e referem queixas quanto aos ambientes de trabalho e insuficiência de materiais e equipamentos.
\end{abstract}

DESCRITORES: Saúde do trabalhador. Terapia ocupacional. Fatores de risco. Ambiente de trabalho/ efeitos adversos. Postura. Condições de trabalho.

\footnotetext{
* Este artigo é baseado no Trabalho de Conclusão de Curso "Identificação de fatores de riscos presentes na prática profissional de terapeutas ocupacionais que atuam junto a portadores de disfunções físicas", desenvolvido pela primeira autora, sob a supervisão do segundo autor junto ao Curso de Terapia Ocupacional da Universidade do Sagrado Coração - USC. Este trabalho foi apresentado no XIV Congresso de Iniciação Científica, 2006, São Carlos. Anais de Eventos da UFSCar, v. 2, p. , 2006, com o título "Identificação de fatores de riscos junto a terapeutas ocupacionais no atendimento de portadores de disfunções físicas".

${ }^{1}$ Terapeuta Ocupacional formada pela Universidade do Sagrado - USC - Bauru.

${ }^{2}$ Docente do Curso de Terapia Ocupacional da Unesp/Marília.

Endereço para correspondência: Nilson Rogério da Silva. Rua: Ana Rosa Zuicker Dannunziatta, 2-27 - Jardim Cruzeiro do Sul. CEP 17030 - 310 - Bauru - SP. e-mail: nilson_ale@ig.com.br
} 
A saúde do trabalhador tem sido foco de discussão em escala mundial, sobretudo em função das doenças de origem ocupacional. Dados do Ministério da Saúde (BRASIL, 2001) revelam índices crescentes de doenças ocupacionais, entre elas: as Lesões por Esforços Repetitivos (LER), também denominados de Distúrbios Ósteo-musculares Relacionados ao Trabalho (DORT), o estresse, a fadiga física e mental e outras manifestações de sofrimento relacionadas ao trabalho.

Pode-se dizer que as doenças ocupacionais estão presentes em todos os segmentos em que a atividade laborativa é realizada. No setor da saúde, o profissional envolvido com o cuidado humano, também tem apresentado altos índices de doenças decorrentes do exercício profissional. Muito embora, teoricamente apresentem o conhecimento técnico para evitar lesões e acidentes, na prática a aplicação destes conhecimentos em benefício próprio nem sempre acontece.

Na literatura é possível encontrar estudos que apontam para a incidência de doenças em profissionais da saúde como: médicos, dentistas, enfermeiros, fisioterapeutas e terapeutas ocupacionais.

Segundo Magalhães et al. (2000) dentre os principais problemas apresentados por médicos-cirurgiões estão a fadiga física e mental, inadequação do ambiente, má postura, repetição de movimentos, acuidade visual diminuída.

Silva (2003) em estudo realizado em uma unidade básica de saúde relata problemas referidos pelos médicos como: salas quentes, mal ventiladas, insuficiência de ventiladores, limitações no espaço físico resultando em dificuldade de transitar pela sala, esforços físicos decorrentes do mobiliário oferecido (macas baixas), materiais obsoletos. Quanto aos dentistas, foram assinalados: falta de materiais disponíveis para o trabalho e utilização de equipamentos antigos, causando aumento do esforço físico (ex: refletor de baixa potência, ausência de brocas).

Em estudo realizado por Guedes et al. (2001) junto a profissionais da enfermagem foram identificados como fatores de risco presentes no trabalho: problemas de ordem psíquica, músculo-esquelético (principalmente coluna) e alterações do sono e vigília.

Silva (2003) aponta sobre os auxiliares de enfermagem: excesso de trabalho impedindo a realização da pausa integral do almoço, dores nas pernas decorrentes da correria na unidade e desgaste mental pelos atritos com os usuários.

Medeiros Neto et al. (2002) em pesquisa realizada junto a fisioterapeutas que atuam com crianças portadoras de problemas neurológicos, identificaram os seguintes problemas: posturas inadequadas adotadas durante manuseio de pacientes, condições precárias dos postos de trabalho (mobiliário, materiais e equipamento, espaço para o trabalho), ausência de condicionamento físico e de pausas atuando como fatores riscos à saúde dos mesmos.

Segundo dados levantados por Morales et al. (1999) junto a terapeutas ocupacionais atuantes na área de disfunções físicas do Hospital Militar Central de Santa Fé de Bogotá, destacam-se fatores de riscos posturais ligados às atividades de tratamento dos pacientes, dimensões limitadas do local de trabalho e permanência prolongada sem alternar posições durante de atendimentos da terapia ocupacional. Neste contexto, é possível observar que a prática dos profissionais de saúde é permeada de situações de riscos à saúde que podem interferir na qualidade da prestação do serviço ao usuário. Nesta pesquisa será enfatizada as condições de trabalho e possíveis implicações para a saúde de terapeutas ocupacionais que atuam na reabilitação física.

Para Gollega et al. (2000) no campo de atuação voltado para a reabilitação física o terapeuta ocupacional:

“... tem por objetivo habilitar ou reabilitar o indivíduo que apresenta uma limitação ou deficiência em seu desempenho, como conseqüência de diferentes condições patológicas, o que interfere direta ou indiretamente em suas atividades cotidianas, tornando-o menos independente" (p. 138).

Na prática é possível observar que o lidar com portadores de disfunções físicas envolve quotidianamente o manuseio do paciente (posicionamento), transferências (cama, cadeira, mudanças de decúbito), locomoção e treino das atividades da vida diária. Esta prática é permeada por atividades que exigem uso da força, posicionamentos durante o manuseio do paciente que exigem esforços posturais do terapeuta (ficar de joelho, agachado, flexão e rotação de tronco). Tais características podem ser agravadas em função da jornada de trabalho e das condições do ambiente e dos materiais e equipamentos utilizados. Não é incomum a prática da terapia ocupacional em reabilitação física ser realizada em locais adaptados, muitas vezes caracterizada por espaços limitados, materiais e equipamentos insuficientes, obsoletos ou inadequados (mesas, macas e tablados com inadequação de altura).

Neste contexto, o terapeuta ocupacional vivencia em seu exercício profissional, condições de trabalho que conduzem a fatores de risco para a sua saúde e que consequentemente podem trazer implicações na qualidade do atendimento prestado ao cliente. Por vezes, a preocupação com o paciente faz com que o terapeuta 
esqueça dos cuidados a serem observados em seu próprio corpo e acaba por adotar posturas lesivas para a estrutura músculo-esquelética, conduzindo às doenças de origem ocupacional.

Outra condição agravante são as práticas relativas ao auto-cuidado e preparação do terapeuta ocupacional para o exercício profissional, ou seja, condicionamento físico (prática de atividades esportivas), alongamento antes e após os atendimentos, realização de pausas etc., que muitas vezes, não faz parte do cotidiano dos profissionais.

No campo da terapia ocupacional há carência de estudos que busquem identificar fatores de riscos presentes na prática profissional. Entretanto, é preciso destacar que para alcançar os objetivos na reabilitação dos pacientes, a atuação do terapeuta ocupacional é permeada por atividades que envolvem uso de força e sobrecarga postural, desgaste físico e mental. Salienta-se que as condições de saúde do terapeuta podem favorecer ou dificultar a eficácia do tratamento prestado ao paciente, bem como potencializar o surgimento de doenças profissionais seja em curto, médio ou longo prazo.

Neste sentido, há necessidade de investigar a dinâmica do trabalho presente na atuação do terapeuta ocupacional a fim de identificar os possíveis riscos existentes para a saúde dos profissionais, bem como propor medidas de prevenção e melhoria das situações de trabalho e consequentemente na assistência aos pacientes.

Esta pesquisa teve por objetivo identificar os principais fatores de riscos presentes na prática profissional terapeutas ocupacionais que atuam na reabilitação de portadores de disfunções físicas, bem como investigar possíveis impactos na saúde.

\section{MÉTODO}

Foram sujeitos da pesquisa 10 terapeutas ocupacionais atuantes na área de reabilitação de portadores de disfunções físicas no município de Bauru e região.

A coleta de dados foi realizada a partir de um roteiro de entrevista contendo 9 questões, sendo algumas abertas e outras fechadas e abordaram os seguintes aspectos: dados pessoais; posições adotadas durante o trabalho; mapeamento de dores e desconfortos; atividades de condicionamento físico; condições do ambiente de trabalho (espaço físico, ventilação, temperatura e iluminação); condições de materiais e equipamentos; sugestões de melhorias para condições de trabalho.

Foi elaborado um termo de consentimento entre pesquisadores e participantes contendo direitos e deveres de ambas as partes. O projeto foi submetido ao Comitê de Ética em Pesquisa (CEP) e aprovado em 22 de setembro de 2005.

\section{Procedimentos da coleta de dados}

Contato telefônico com os terapeutas ocupacionais nos seus locais de trabalho a fim de explicar os objetivo da pesquisa e convidá-los a participar da mesma.

- Agendamento do participante por contato telefônico para realização da entrevista em local que considerado mais adequado. Antes da entrevista a pesquisadora fez uma apresentação do roteiro de entrevista.

\section{Procedimentos de análise dos dados}

Após a realização das entrevistas, as informações foram transcritas e os discursos categorizados e organizados em figuras e tabelas.

\section{RESULTADOS E DISCUSSÕES}

Os resultados da pesquisa serão apresentados de acordo com as categorias investigadas no roteiro de entrevista, conforme a seguir:
A) Dados demográficos;
B) Principais posições adotadas no trabalho;
C) Principais regiões e desconfortos;
D) Afastamento do trabalho;
E) Atividades de condicionamento físico;
F) Preparação antes de iniciar o trabalho;
G) Pausas durante o trabalho;
H) Cuidado durante o manuseio dos pacientes;
I) Ambiente e posto de trabalho;
J) Sugestões para a melhoria do trabalho.

\section{A) Dados demográficos}

Participaram da pesquisa 10 terapeutas ocupacionais, todos do sexo feminino atuantes em Bauru e região. As

\footnotetext{
(1) Foi solicitado junto ao CREFITO o número de terapeutas ocupacionais atuantes na região, bem como uma forma para contactá-los. Entretanto, não obteve-se resposta em tempo hábil e desta forma, foi feito levantamento junto as instituições públicas e particulares da cidade e região via contato telefônico para compor a amostra).
} 
idades variam entre 25 anos a 48 anos e quanto ao tempo de formado de 6 meses a 25 anos. Todos os sujeitos trabalham junto a portadores de disfunções físicas em instituições (associações, hospitais e clínicas) realizando em média 30atendimentos semanais (individuais e em grupo).

\section{B) Principais posições adotadas no trabalho}

As principais posturas adotadas pelos terapeutas ocupacionais foram objeto de investigação, sendo possível observar que a posição predominantemente adotada durante o trabalho entre terapeutas ocupacionais é a sentada com 9 ocorrências, seguida da posição em pé (8), sentado com o tronco flexionado (7), em pé com o tronco flexionado (5).

De acordo com Coury (1995) a postura sentada pode ocasionar um aumento da pressão no interior do disco intervertebral, achatamento do arco lombar, dores na região lombar (lombalgias) e discopatias como a hérnia de disco.

Tabela 1 - Posições adotadas durante o trabalho.

\begin{tabular}{l|c}
\hline Posições adotadas & $\mathbf{N}^{\mathbf{0}}$ de ocorrências \\
\hline Sentado & 9 \\
Sentado com o tronco flexionado & 7 \\
Em pé com os joelhos flexionados & 3 \\
Em pé com o tronco flexionado & 5 \\
Ajoelhado & 3 \\
Agachado & 1 \\
Em pé & 8 \\
Andando & 4 \\
Ajoelhado com o tronco para frente & 1 \\
\hline
\end{tabular}

\section{C) Principais regiões e desconfortos}

Os profissionais foram questionados quanto aos principais sintomas presentes e quais os segmentos corporais envolvidos durante o exercício profissional. Os resultados indicam que a dor é o desconforto predominante (31 ocorrências) sendo que a região corporal de maior incidência são os membros superiores com 17 ocorrências, seguida de coluna (9).

Coury (1995) destaca que quando há utilização intensa de membros superiores, os tendões sofrem atrito entre o osso e o ligamento e com a repetição dos movimentos amplos (maiores que $60^{\circ}$ ) ocorre a inflamação dos tendões, facilitando o aparecimento de tendinites.

Neste estudo pode-se destacar que o trabalho dos terapeutas ocupacionais, por vezes, comporta movimentos amplos de ombros associados com o emprego de força favorecendo o desenvolvimento de processo inflamatório como tendinites e bursites. Os movimentos extremos da coluna, bem como posturas extremas do tronco com impacto para a coluna, acompanhados do uso de força também merecem consideração por estarem presentes durante o manuseio de pacientes com disfunções físicas, o que pode favorecer o surgimento de lombalgias e discopatias.

\section{D) Afastamento do trabalho}

Nesse item, observa-se que apenas $10 \%$ dos entrevistados apresentaram absenteísmo (10 dias de afastamento) determinado exercício profissional, neste caso estando relacionados a problemas na coluna. A maioria (90\%) dos profissionais não se ausentaram do trabalho.

\section{E) Atividade de condicionamento físico}

Sobre a realização de atividades que promovem o condicionamento físico, $70 \%$ dos terapeutas entrevistados relataram que não fazem modalidade alguma; $30 \%$ disseram que realizam ginástica localizada, alongamentos e caminhada pela manhã.

Segundo Anderson (1998), alongar-se durante o dia pode ajudar a prevenir a rigidez e dor muscular, melhorar a circulação, diminuir a ansiedade, estresse e fadiga, diminuição de riscos de lesão.

Desta forma, pode-se apontar o baixo índice de envolvimento dos participantes da pesquisa (30\%) em atividades que atuam de maneira preventiva e promovem a saúde diminuindo o riscos de lesões e doenças ocupacionais.

\section{F) Preparação antes de iniciar o trabalho}

A pesquisa aponta que $90 \%$ dos terapeutas não realizam atividades de preparação física antes de iniciar o trabalho, relataram não possuir esse hábito, enquanto $10 \%$ disseram que às vezes procuram fazer alongamentos. Anderson (1998) salienta a necessidade de investimento na preparação corporal antes de iniciar o trabalho como forma de aliviar a tensão.

\section{G) Pausas durante o trabalho}

Segundo Couto (1996), o intervalo de 15 minutos no período da manhã e da tarde atendem aos seguintes objetivos: ajuda a prevenir a fadiga; fornece oportunidade para reduzir o grau de tensão; oferece oportunidade para alguma interação social. As pausas quando prescritas em quantidade e duração adequadas reduzem o absenteísmo, uma vez que a mesma representa a possibilidade e necessidade do descanso.

Nesta pesquisa, $80 \%$ dos entrevistados responderam 
que realizam pausas durante o trabalho, enquanto apenas $20 \%$ disseram que não. Dos participantes que responderam sim, $10 \%$ descansam 1 hora, $20 \% 10$ minutos e 50\% 15 minutos. Com relação ao tempo de duração das pausas a resposta predominante foi de 15 minutos, sendo realizada principalmente após 3 horas de trabalho.

Sobre este aspecto, salienta-se que a maioria dos profissionais procuram realizar as pausas e portanto atuam de forma a permitir repouso do corpo prevenindo a fadiga muscular e os riscos de lesões.

\section{H) Cuidados durante manuseio de pacientes}

Em relação aos cuidados adotados pelos terapeutas durante o manuseio dos pacientes, $90 \%$ responderam que procuram prestar atenção nos atendimentos, enquanto $10 \%$ não se preocupam com essa temática. Entre os cuidados adotados destacam-se: corrigir posturas durante o atendimento; evitar sobrecargas musculares e movimentos desnecessários; cuidados ao manusear crianças pesadas; solicitar colaboração de outro terapeuta para ajudar nas transferências de pacientes; diminuir esforços dos pacientes.

Por outro lado, as respostas dos participantes manifestam contradição quando consideramos o número significativo de referências de dores na coluna, membros superiores e inferiores, necessitando de maior investigação e adoção de outras medidas de controle da coleta de dados, como por exemplo fotografar ou filmar as situações de trabalho. Tal questionamento pode indicar uma não percepção dos profissionais em relação às posturas adotadas durante o trabalho, sobretudo no manuseio dos pacientes.

\section{I) Ambiente e posto de trabalho}

Os resultados mostram que $60 \%$ dos terapeutas ocupacionais consideram adequado o espaço físico para o desenvolvimento do trabalho, enquanto $40 \%$ parcialmente adequado. Entre os problemas identificados estão: espaço físico deveria ser maior, devido à demanda de pacientes ser grande e falta de mobiliários adequados.

$\mathrm{Na}$ avaliação do ambiente, $80 \%$ dos participantes acreditam que a ventilação está adequada e $20 \%$ parcialmente adequada. Em relação à temperatura $60 \%$ avaliam como adequada e $40 \%$ parcialmente adequada. Quanto à iluminação, $80 \%$ responderam como adequada e $20 \%$ parcialmente adequada. Os principais problemas identificados foram: número insuficiente de lâmpadas; pouca ventilação; sala escura; calor intenso; número insuficiente de ventiladores; falta de planejamento do espaço físico; falta de iluminação; falta de ventilação natural.
Ainda que a maioria dos participantes tenha avaliado os locais de trabalho de forma positiva, é preciso considerar que as queixas expressas acima denotam preocupação e necessidade de melhorias nas condições ambientais, uma vez que como sugere Delboni (1997) a presença de ambientes claros, ventilados e iluminados, aliados à oferta de mobiliários adequados, favorece melhor desempenho das funções.

Seligmann (1994) alerta para a presença de calor, ruídos, vibrações entre os fatores ambientais que podem afetar a saúde dos trabalhadores.

A presença de problemas nas instalações de unidade e serviços de saúde é um tema recorrente, Galdino e Soares (2002), ao estudarem um hospital público em Recife identificaram a presença ambientes inadequados, salas e locais de trabalho improvisados.

No que se refere à distribuição de materiais e equipamentos nas salas de atendimentos, $60 \%$ dos entrevistados responderam que consideraram adequada, $30 \%$ parcialmente adequada e 10\% inadequada. Foram citados como problemas: falta de recursos financeiros das instituições para aquisição de materiais e equipamentos; falta de materiais de consumo; aglomeração de materiais devido a limitação do espaço físico.

Quanto aos materiais e equipamentos disponibilizados para o exercício profissional, $60 \%$ dos terapeutas responderam que são suficientes, enquanto $40 \%$ insuficientes, sendo identificados as seguintes queixas: falta de materiais para confecção de órtese (talas); falta ou insuficiência de materiais para realização de exercícios (massas de silicone, redes de força); falta ou insuficiência de mesas para realização das atividades; falta de equipamentos para avaliação e atividades funcionais; falta de materiais para estimulação precoce com bebês.

Silva (2003) ao estudar uma Unidade Básica de Saúde no interior de São Paulo verificou a presença de equipamentos obsoletos que dificultavam o trabalho e geravam sobrecarga de trabalho nos trabalhadores favorecendo o surgimento de doenças ocupacionais.

Com relação à conservação dos materiais e equipamentos $100 \%$ dos entrevistados responderam que estão em bom estado.

\section{J) Sugestões para a melhoria do trabalho}

Foi solicitado aos participantes da pesquisa que apontassem sugestões para a melhoria das condições de trabalho e manutenção da saúde. As principais manifestações expressas foram:

Materiais e equipamentos: necessidade de materiais para confecções de órteses (talas); disponibilizar materiais 
mais adequados (mesas para adultos e crianças); introdução de materiais e equipamentos com alta tecnologia para facilitar a atuação profissional e desempenho do paciente;

Espaço físico: necessidade de local reservado para terapia ocupacional; maior privacidade nos atendimentos individuais (salas compartilhadas); planejamento do espaço físico (participar do planejamento).

Inserção: criar mecanismos que permitam a inserção de pacientes no mercado de trabalho frente às possibilidades das cidades.

Formação e capacitação profissional: Integração entre teoria e prática; oferecer atualização profissional por meio de cursos, congressos, pesquisas.

Reconhecimento e autonomia profissional: $n$ ecessidade de maior autonomia profissional; divulgação da terapia ocupacional junto a outros profissionais; conquistar espaço de trabalho e reconhecimento.

\section{CONSIDERAÇÕES FINAIS}

De maneira geral é possível concluir que as condições de trabalho oferecidas para o desenvolvimento da atividade de terapeutas ocupacionais junto a portadores de disfunções físicas, podem conduzir ao processo de adoecimento. Tal fato pode ser demonstrado a partir dos dados levantados na pesquisa que indicam a predominância da postura em pé, sentada e posições que envolvem a flexão de tronco. A alternância de posições adotadas durante $\mathrm{o}$ trabalho pode ser entendida como fator positivo na medida em que evita sobrecarga em algumas região corporal, permitindo o uso de grupos musculares variados. Por outro lado, a flexão excessiva de tronco pode conduzir a problemas na coluna como lombalgias e discopatias, podendo assim ser estabelecida uma relação com a incidência de dores na coluna (9 ocorrências). O índice significativo de dores nos mesmos superiores (17 ocorrências) pode estar relacionado ao uso de força bastante presente durante o manuseio de pacientes.

Como agravante das condições mencionadas acima, merece destaque o baixo índice de adesão às atividades de condicionamento físico (apenas 30\% realizam), bem como o fato de um número restrito de terapeutas ocupacionais $(10 \%)$ realizarem atividades de preparação para o trabalho, como por exemplo, o alongamento. Tais medidas poderiam atenuar a carga de trabalho e diminuir os impactos sobre os segmentos corporais.

A realização de pausas durante o trabalho pela maioria dos entrevistados ( $80 \%$ ) e a adoção de cuidados durante o manuseio de pacientes $(90 \%)$ podem ser apontados como fatores positivos, uma vez que diminuem os riscos de doenças ocupacionais. Salienta-se que as condições de saúde dos profissionais podem gerar impacto na qualidade dos serviços prestados aos clientes.

Em relação ao ambiente e posto de trabalho foram investigados os seguintes aspectos: espaço físico, ambiente (ventilação, temperatura e iluminação), distribuição de materiais e equipamentos nas salas de atendimento, materiais disponibilizados para o exercício profissional. Em todos os aspectos investigados, a maioria dos participantes considerou adequada. Entretanto, algumas sugestões para melhoria das condições de trabalho foram apresentadas pelos participantes, entre elas: necessidade de ampliação do espaço físico frente à demanda de pacientes, aquisição de mobiliários adequados, aumentar a qualidade da iluminação (número insuficiente de lâmpadas, salas escuras), investimento em melhoria da ventilação, melhoria das condições térmicas (presença de calor intenso), investimento em recursos financeiros para compra de materiais e equipamentos.

Assim, pode-se concluir que as condições oferecidas para o exercício profissional dos terapeutas ocupacionais, bem como algumas condutas inadequadas adotadas durante o trabalho favorecem o surgimento de doenças ocupacionais, sobretudo para a estrutura músculoesquelética.

Neste sentido, indicam a necessidade de intervenção no ambiente e posto de trabalho para eliminação de fatores de risco, bem como de programas junto aos terapeutas como forma de trabalhar a prevenção e promoção da saúde por meio da adoção de auto-cuidados. Desta forma, é possível caminhar para melhoria das condições de trabalho e da saúde dos terapeutas ocupacionais e consequentemente na qualidade dos serviços prestados à clientela.

Este pesquisa traz contribuições de um estudo piloto para a compreensão das condições de trabalho e saúde de terapeutas ocupacionais que atuam na reabilitação. Entretanto, salienta-se a necessidade estudos que abranjam um percentual maior de profissionais a fim de que os dados possam ser mais universalizáveis. Assim, é preciso considerar na coleta de dados outras fontes, como por exemplo, fotografia e filmagem das situações de trabalho, como maneira de somar-se ao relato verbal dos profissionais envolvidos.

Segundo Lancman e Jardim (2004) no conjunto de ações da prática em Terapia Ocupacional em saúde e trabalho estão a prevenção, o tratamento, a reabilitar e reinserção dos indivíduos no trabalho. Ressalta ainda a necessidade de transformar “... os processos ativos para que os indivíduos 
possam refletir sobre o próprio trabalho para mudar sua relação com o mesmo" (p. 89).

Contudo, esta transformação precisa ser difundida a partir do olhar sobre a própria profissão, visto que a melhoria das condições de trabalho e saúde dos terapeutas ocupacionais guarda estreita relação com as possibilidades de sucesso na eficácia junto aos mais diferentes indivíduos ou situações de trabalho.

Espera-se que este estudo possa contribua para o surgimento de novas pesquisas abordando esta temática ainda pouco estudada entre os terapeutas ocupacionais.

CRUZ, J. P.; SILVA, N. R. Identification of factors of risks in occupational therapists in the attendance of carriers of physical dysfunctions. Rev. Ter. Ocup. Univ. São Paulo, v. 18, n. 1, p. 22-29, jan./abr., 2007.

\begin{abstract}
Data obtained from the Ministry of Health (Brazil, 2001) reveal growing rates of occupational illnesses among all career segments. The professional involved with the care of the human being within the health sector have also been presenting high rates of health problems resulted from the job performance. This research attempted to investigate the working conditions of the Occupational Therapists that deal with physically disabled people in order to identify some of the risk factors of the job, possible impacts in their health, as well as suggest prevention measures and improvement of working situations. These conditions will than reflect on the treatment of the patients. The sample was composed by 10 Occupational Therapists from the city of Bauru and region. The data was collected based on a structured interview questionnaire, which was entirely transcripted after the data gathering. The answers were than categorized and organized into figures and tables. It was possible to conclude that the working conditions presented to the Occupational Therapists can lead to a development process of illness. The activities are mainly realized on either standing or seating positions, improper column postures during the work performance with the patients, in addition to the employment of strength. There were also reports of low rates of physical conditioning activities and breaks taken during the job, as well as complaints related to the work environment, and lack of material and equipment.
\end{abstract}

KEY WORDS: Occupastional health. Occupational therapy. Risk factors. Working environment/ adverse effects. Posture. Working conditions.

\title{
REFERÊNCIAS
}

ANDERSON, B. Alongue-se no trabalho. São Paulo: Summus, 1998.

BRASIL. Ministério da Saúde do Brasil. Organização PanAmericana da Saúde no Brasil. Doenças relacionadas ao trabalho: manual de procedimentos para os serviços de saúde. Brasília, D.F., 2001. (Série A. Normas e manuais técnicos; n. 114).

CARLO, M. M. R. P; BARTALOTTI, C. C. (Orgs.). Terapia ocupacional: fundamentos e prática. São Paulo: Plexus, 2001. p.137-154.

COURY, H. J. C. G. Trabalhando sentado - manual para posturas sentadas confortáveis. 2a ed. São Carlos: Ed. UFSCar, 1995.

COUTO, H. A. Ergonomia aplicada ao trabalho VII. Manual técnico da máquina humana. Belo Horizonte: Ergo, 1996. v.2.

DELBONI, T. H. Vencendo o stress. São Paulo: Makon Books, 1997.
GALDINO, A. S.; SOARES, M. M. A influência do ambiente hospitalar na qualidade dos serviços de saúde: uma análise nos setores de internamento pediátrico em hospitais públicos em Recife/PE. Recife, 2002.

GOLLEGA, A. C. C.; LUZO, M. C. M.; DE CARLO, M. M. R. P. Terapia ocupacional - princípios, recursos e perspectivas em reabilitação física. In: DE GUEDES, E. M; MAURO, M. Y. C.; MAURO, C. C. C.; MORIYA, T. M. Problemas músculoesqueléticos na enfermagem hospitalar. In: CONGRESSO BRASILEIRO DE ERGONOMIA, 10., PAN AMERICANO DE ERGONOMIA - ABERGO, Rio de Janeiro, 2000. Anais. Rio de Janeiro: ABERGO, 2000.

HAGEDORN, R. Fundamentos da prática da terapia ocupacional. São Paulo: Dynamis, 2001.

LANCMAN, S.; JARDIM, T. A. O impacto da organização do trabalho na saúde mental: um estudo em psicodinâmica do trabalho. Rev. Ter. Ocup. Univ. São Paulo, v. 15, n. 2, 2004, p. 82-89. 
CRUZ, J. P.; SILVA, N. R. Identificação de fatores de riscos. Rev. Ter. Ocup. Univ. São Paulo, v. 18, n. 1, p. 22-29, jan./abr., 2007.

MAGALHÃES, R. A. S.; CARVALHO, D. T.; MÁSCULO, F. S. Identificação de riscos ergonômicos no posto de trabalho de médicos-cirurgiões em um hospital universitário. In: CONGRESSO BRASILEIRO DE ERGONOMIA, 10.; PAN AMERICANO DE ERGONOMIA - ABERGO, Rio de Janeiro, 2000. Anais. Rio de Janeiro: ABERGO, 2000. p.22-23.

MEDEIROS NETO, C. F.; CÂMARA, M. R.; OLIVEIRA, S. C. F. Avaliação da biomecânica ocupacional dos fisioterapeutas neuro-pediatras: um estudo de caso. In: CONGRESSO LATINO-AMERICANO DE ERGONOMIA, 12.; CONGRESSO BRASILEIRO DE ERGONOMIA, 12.; SEMINÁRIO BRASILEIRO DE ACESSIBILIDADE INTEGRAL-ABERGO, 2002. Anais.
MORALES, A. A. M; QUIÑONES, M. E. Q.; CRUZ, M. L. T.

Factores de riesgos ergonômicos a los que vem expuestos los Terapeutas ocupacionales que se desempeñan em el área de discapacidades físicas Del Hospital Militar Central de Santa Fé de Bogotá. Rev. Ocup. Humana, v. 8, n. 2, p. 32-42, 1999.

SELIGMANN, S. E. Desgaste mental no trabalho dominado. Rio de Janeiro: UFRJ/Cortez, 1994.

SILVA, N. R. Estudo das condições de trabalho em unidade básica de saúde no município de São Carlos: a perspectiva dos diferentes autores. 2003. Dissertação (Mestrado - Engenharia de Produção) Universidade Federal de São Carlos, São Carlos, 2003.

Recebido para publicação: Mar./2007

Aceito para publicação: Abr./2007 